\title{
Specificity Protein 1 Regulates Gene Expression Related to Fatty Acid Metabolism in Goat Mammary Epithelial Cells
}

\author{
Jiangjiang Zhu ${ }^{1, \dagger}$, Yuting Sun ${ }^{1, \dagger}$, Jun Luo ${ }^{1, *}$, Min Wu ${ }^{1}$, Jianhua Li ${ }^{1}$ and Yanhong Cao ${ }^{2}$
}

1 Shaanxi Key Laboratory of Molecular Biology for Agriculture, College of Animal Science and Technology, Northwest A\&F University, Yangling 712100, China; E-Mails: zhujiang4656@hotmail.com (J.Z.); yutingsun2008@126.com (Y.S.); minwu1229@163.com (M.W.); lijianhua991@gmail.com (J.L.)

2 Guangxi Institute of Animal Husbandry, Guangxi Zhuang Autonomous Region, Nanning 530000, China; E-Mail: caoyh610@163.com

$\dagger$ These authors contributed equally to this paper.

* Author to whom correspondence should be addressed; E-Mail: luojun@nwsuaf.edu.cn; Tel.: +86-29-8708-2891; Fax: +86-29-8708-2892.

Academic Editor: Mark L. Richter

Received: 5 December 2014 / Accepted: 6 January 2015 / Published: 14 January 2015

\begin{abstract}
Specificity protein 1 (SP1) is a ubiquitous transcription factor that plays an important role in controlling gene expression. Although important in mediating the function of various hormones, the role of SP1 in regulating milk fat formation remains unknown. To investigate the sequence and expression information, as well as its role in modulating lipid metabolism, we cloned SPI gene from mammary gland of Xinong Saanen dairy goat. The full-length cDNA of the SP1 gene is $4376 \mathrm{bp}$ including $103 \mathrm{bp}$ of 5'UTR, $2358 \mathrm{bp}$ of ORF (HM_236311) and 1915 bp of 3'UTR, which is predicted to encode a 786 amino acids polypeptide. Phylogenetic tree analysis showed that goat SP1 has the closest relationship with sheep, followed by bovines (bos taurus, odobenus and ceratotherium), pig, primates (pongo, gorilla, macaca and papio) and murine (rattus and mus), while the furthest relationship was with canis and otolemur. Expression was predominant in the lungs, small intestine, muscle, spleen, mammary gland and subcutaneous fat. There were no significant expression level differences between the mammary gland tissues collected at lactation and dry-off period. Overexpression of $S P 1$ in goat mammary epithelial cells (GMECs) led to higher mRNA expression level of peroxisome proliferator-activated receptor- $\gamma(P P A R \gamma)$ and lower liver $X$ receptor $\alpha(L X R \alpha)$ mRNA level, both of which were crucial in regulating fatty
\end{abstract}


acid metabolism, and correspondingly altered the expression of their downstream genes in GMECs. These results were further enhanced by the silencing of SP1. These findings suggest that SP1 may play an important role in fatty acid metabolism.

Keywords: dairy goat; mammary epithelial cells; specificity protein 1; fatty acid metabolism

\section{Introduction}

The importance of goats as essential food in meat and dairy industries has been discussed and documented [1]. High contents of beneficial fatty acids endow goat milk with a high nutritional value, which also prevents some human metabolic disorders [1,2]. Although several genes including peroxisome proliferator-activated receptor- $\gamma(P P A R \gamma)$ [3], sterol regulatory element binding proteins 1 (SREBP1) [4] and liver X receptor $\alpha(L X R \alpha)$ [5], have been reported to be crucial in regulating milk fat metabolism, the regulatory mechanisms of lipid metabolism remain unclear.

Specificity protein 1 (SP1), a member of Sp/Kruppel super family [6], is important for transcription of many cellular and viral genes that contain GC boxes in their promoters [7], particularly to those genes related to biosynthesis and metabolism of nucleic acids (e.g., thymidylate synthase, adenine deaminase and DNA polymerase), cell cycle and proliferation [8]. It regulates the expression of genes by binding to the GC-rich domains (GGGGCGGGG/GGTGTGGG) in gene promoters to enhance transcription $[8,9]$. In the absence of a TATA-element, SP1 also facilitates a sequential recruitment of transcription factor IID (TFIID) and RNA polymerase II [10-12]. Based on this evidence, SP1 is well known as a basal transcription factor [6].

Increasing reports are revealing that SP1 may be involved in lipid metabolism regulation. SP1 has been shown to be one of the major transcription activators of FASN [13], the crucial enzyme for de novo fatty acid synthesis. In breast cancer cells the silencing of SPI expressed significantly lower levels of FASN and SREBP-1c mRNA [14]. Similarly, SP1 overexpression enhanced the expression of human FASN in the presence of SREBP-1c [13]. To the contrary, decreased FASN-promoter activity was observed without the help of SREBP1 in rat hepatocytes [15]. It is a fact that, 34\% of SREBP1 targets are occupied by SP1, which is partly explained by synergistic action, [16] such as, ATP-citratelyase (ACLY) and acetyl-CoA carboxylase (ACACA) both of which are downstream of SREBP1 [17] and contain SP1 binding sites in the promoters [18,19]. Besides, SP1 functions as a co-factor of signal transducer and activator of transcription 3 (Stat3) [7], activates the promoter of acyl-coenzyme-A oxidase (ACOX), the rate-limiting enzyme in peroxisomal beta-oxidation of fatty acids, in synergy with peroxisome proliferator-activated receptor (PPAR $\gamma$ ) or retinoid-X receptor (RXR) [20]. Moreover, SP1 and hypoxia-induced factor 1 (HIF1) were also reported to co-regulate the expression of ATP-binding cassette A1 (ABCA1), which is one of the major regulators in mediating cellular efflux of phospholipids and cholesterol [21]. Overall, these literatures suggest that SP1 may also play an important role in regulating expression of genes involved in lipid metabolism. Despite the importance of SP1, lack of sequence and expression information hindered functional research in dairy goats. 
In the present study, we cloned the full-length cDNA of the SP1 gene from mammary gland of Xinong Saanen dairy goats and analyzed the sequence by bioinformatics. A tissue extensive expression distribution was identified. The effect of SP1 expression levels on the expression of PPAR $\gamma, S R E B P 1$ and $L X R \alpha$ were investigated in goat mammary epithelial cells (GMECs) with adenovirus-mediated overexpression and siRNA-mediated RNA interference respectively. These basal data may provide original sequence and expression information of the $S P 1$ gene and is also better for our understanding about the regulatory role of $S P 1$ in milk fat formation in mammary gland of dairy goats.

\section{Results}

\subsection{Characterization of SP1 cDNA from Goat Mammary Gland}

The full-length cDNA of SP1 gene is 4376 bp, includes 103 bp of 5'UTR, 2358 bp of ORF (HM_236311) and $1915 \mathrm{bp}$ of 3'UTR, predicted to encode a 786 amino acids polypeptide. An ATG initiation codon is located at $104 \mathrm{nt}$, and a TAA stop codon is situated at $2462 \mathrm{nt}$. The sequence also contains one AACAAA motifs, which represent putative polyadenylation signals (nt3893-3898) (Figure S1). The coding sequence (CDS) of goat SP1 shares 99.6\%, 94.7\% and 91.9\% similarity with bovine (NM_001078027.1), human (NM_138473.2), and rattus (NM_013672.2) respectively. Correspondingly, the similarity of the amino acid sequence is $99.3 \%, 96.9 \%$ and $95 \%$, respectively. The similarity of 5 'UTR is $100 \%, 97.6 \%$ and $96.8 \%$, and that of the 3 'UTR is $97 \%, 86 \%$ and $86 \%$, with previously indicated species, respectively (Figure S2).

The SP1 protein has a calculated molecular weight of 80,840.1-Da and isoelectric point (PI) of 7.23. The most abundant amino acid is serine (Ser), which contains up to 100 amino acids, accounts for $12.7 \%$ of total amino acids. It contains 41 negatively charged residues (Asp and Glu) and 41 positively charged residues (Arg and Lys). The estimated half-life of the protein is $30 \mathrm{~h}$ in mammalian reticulocytes in vitro, $20 \mathrm{~h}$ in yeast in vivo and $10 \mathrm{~h}$ in Escherichia coli, respectively. This protein is computed to be unstable with 52.26 of instability index (II) and 68.80 of aliphatic index. The grand average of hydropathicity (GRAVY) is -0.440 , max at 1.978 and min at -3.2 (Figure $\mathrm{S} 1$ ).

Before the $N$-terminus Met1...Gly29 residues, is a signal peptide with no transmembrane helix. An acetylation site is predicted at Ser2, and three zinc fingers are predicted in Cys529...His551, Cys559...His581 and Cys589...His709, respectively. The SP1 protein contains 45 serine phosphorylation sites, 9 threonine phosphorylation sites, 3 tyrosine phosphorylation sites and 11 glycosylation sites. In addition, the SP1 protein contains 2 GLN rich regions (Gln156...Gln213 and Gln352...Gln481) and 1 SRE-rich region (Ser271...Ser328) (Figure S1).

We also investigated the secondary structure and compared the tertiary structure of SP1 protein of capra hircus, bovine, human, and mouse. The protein of SP1 contains 3 alpha helices-rich regions (Figure 1A). The spatial structure of SP1 protein shares a high similarity among different species (Figure 1B). 


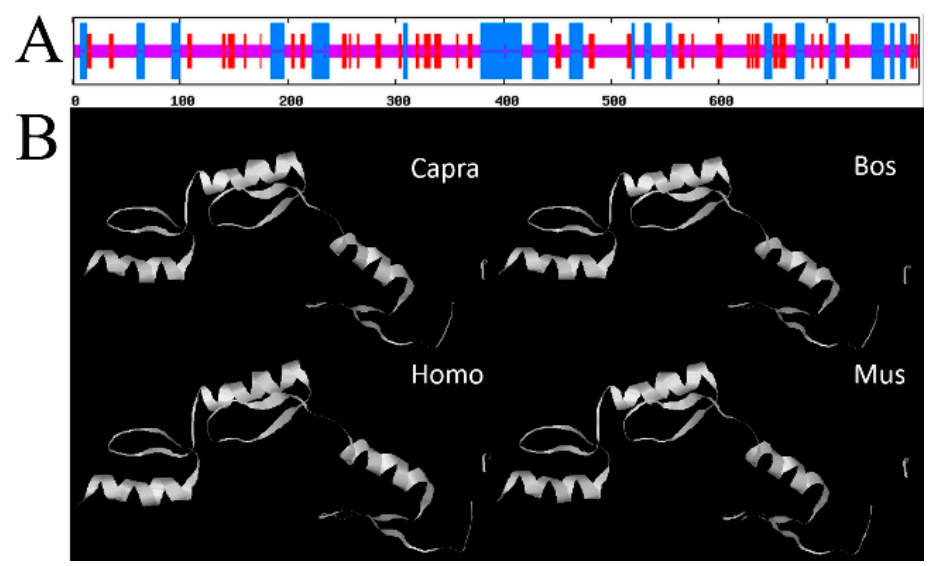

Figure 1. Structure prediction of SP1 proteins. (A) The secondary structure of SP1 protein. Blue color represents alpha helixes, red color represents beta bridges, and purple represents random coils; (B) The tertiary structure of SP1 protein in various species.

\subsection{Phylogenetic Analysis Based on SP1 Amino Acid Sequences}

To investigate potential evolutionary processes of the SPl gene among various species, a neighbor-joining phylogenetic tree was constructed, based on sequences of 31 representative animals. The result shows that goat SP1 has the closest relationship with sheep, followed by bovines (including bos taurus, odobenus and cerato therium), pig, primates (pongo, gorilla, macaca and papio) and murine (rattus and mus). Canis and otolemur show the greatest distance from capra hircus (Figure 2).

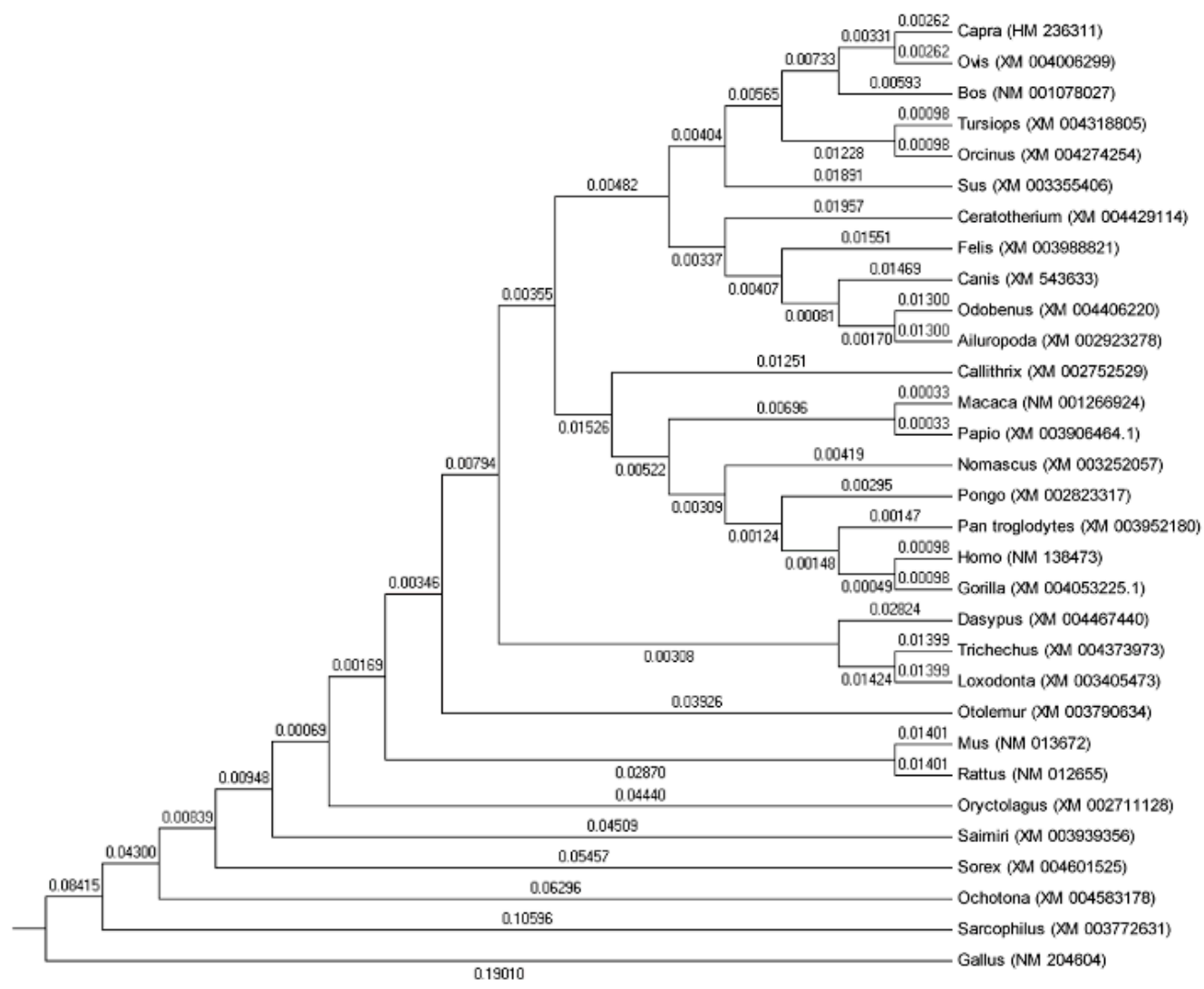

Figure 2. Phylogenetic tree based on $S P 1$ gene sequences of 31 representative animals made with MEGA 5 software (S. Kumar, Tempe, AZ, USA) using Neighbor-Joining (NJ) method. 


\subsection{Analysis of the Expression Profile of Goat SP1}

In order to enhance understanding the role of $S P 1$ in various tissues of Xinong Saanen dairy goat, we investigated the mRNA expression profiles of SP1. SP1 was expressed at highest levels in lungs and small intestine, followed by muscle, spleen, mammary gland and subcutaneous fat tissues. Stomach, kidney and liver showed similar SP1 expression levels. Heart had the lowest expression mRNA level among the tissues examined (Figure 3A).

To investigate whether the expression was affected by different lactation stages, we assayed SP1 mRNA level from the tissue samples in the lactation and dry-off periods. However, we did not find any significant expression differences between the two periods, though a tendency of higher expression was observed from lactation samples (Figure 3B).
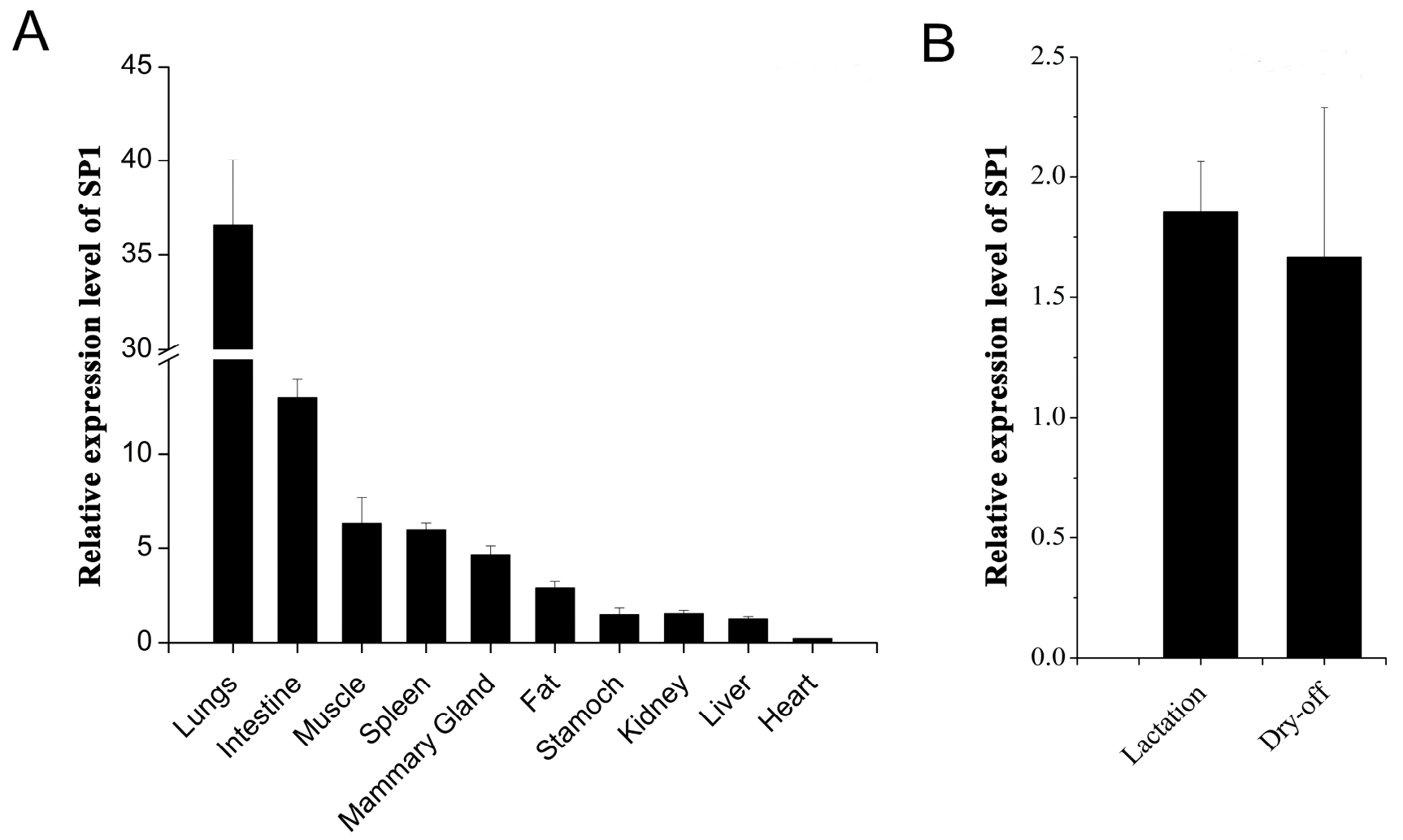

Figure 3. The mRNA expression profiles of $S P 1$. (A) mRNA expression of $S P 1$ in various tissues in dairy goats; (B) Expression level of SP1 between lactation and dry-off periods.

\subsection{SP1 Is Involved in Lipid Metabolism Regulation}

After treatment with adenovirus of Ad-SPl for $72 \mathrm{~h}$, both the protein (Figure 4A) and mRNA expression (Figure 4B) of SP1 were significantly increased. Accompanying the overexpression of the $S P 1$ gene, an obvious increase was observed in PPAR $\gamma$ expression, while $L X R \alpha$ was decreased dramatically by adenovirus treatment (Figure 4C). Corresponding to the result of $S P 1$ overexpression, silencing of $S P 1$ (Figure 4D,E) decreased the expression of PPAR $\gamma$ and increased the expression of $L X R \alpha$ significantly (Figure 4F). However, SREBPI remained unaffected after adenovirus or siRNA treatments, though a slight decrease was observed (Figure 4C,F). 
Relative to the control (Scramble, negative siRNA), silencing of $S P 1$ markedly decreased the expression of CD36 molecule (CD36), acyl-CoA synthetase long-chain family member 1 (ACSL1) and heart type fatty acid bind protein (FABP3). All of these results were enhanced by the increased expression of these genes in Ad-SP1 treatment. The expression of $A C A C A$ was increased by the loss of SP1 and decreased in adenovirus treatment. The expression of lipoprotein lipase $(L P L)$ was upregulated with the overexpression of SP1, but not changed significantly by the reduction of SP1. Conversely, while FASN and cytosolic acetyl-CoA synthase (ACSS2) were not affected by Ad-SP1 treatment relative to the control (Ad-GFP), the loss of SP1 reduced their mRNA content markedly.
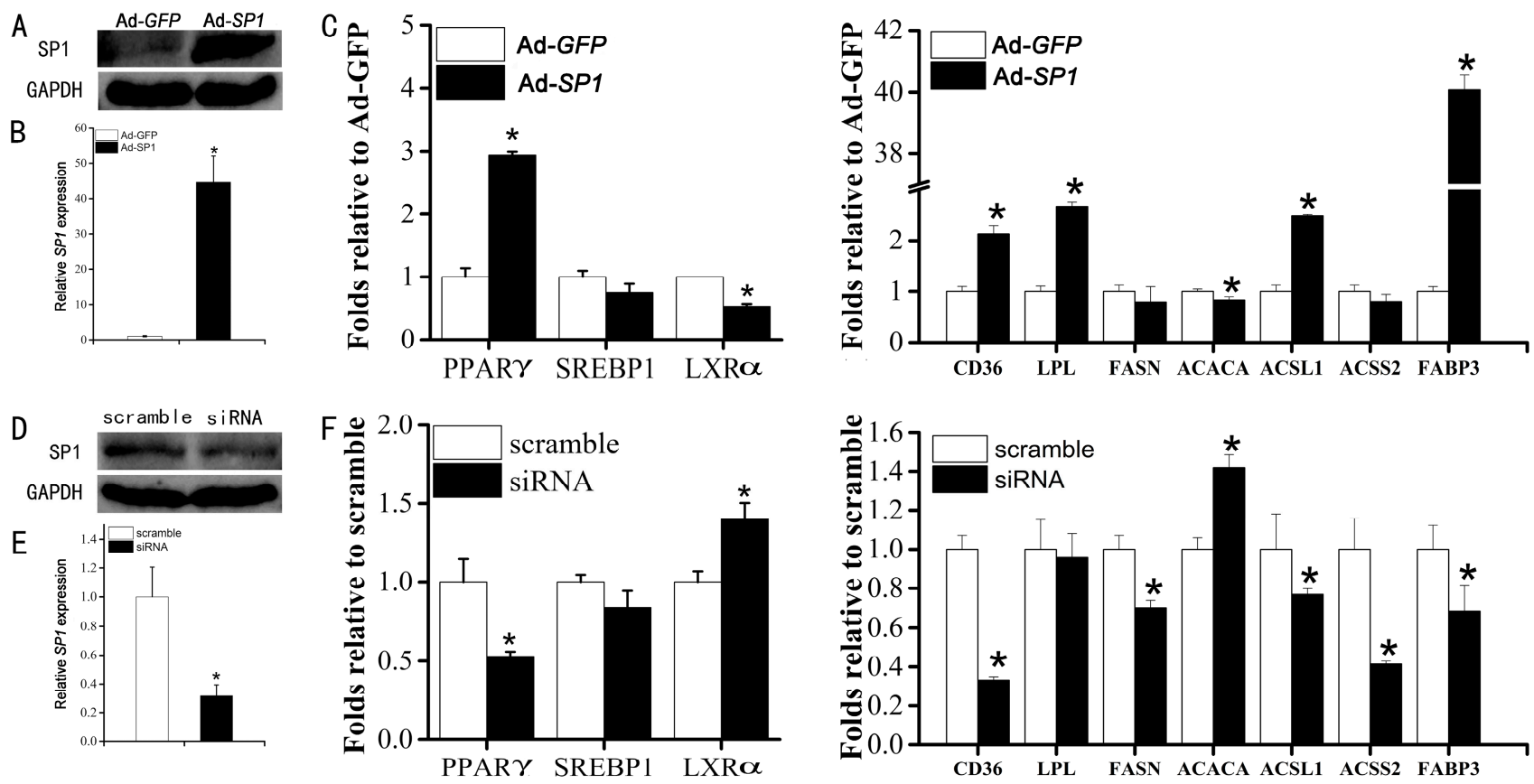

Figure 4. Effect of expression alteration of SP1 on the expression of genes relative to lipid metabolism. (A) Ad-SP1 overexpressed the expression level of SP1 protein; (B) Ad-SP1 overexpressed the mRNA expression level of the $S P 1$ gene; (C) Overexpression of $S P 1$ altered the mRNA expression level of genes related to lipid metabolism in goat mammary epithelial cells (GMECs); (D) siRNA decreased the protein expression of SP1; (E) siRNA decreased the mRNA expression of $S P 1$; and (F) The silencing of SP1 affected the mRNA expression of genes associated with lipid metabolism. Columns, average of 3 repeats; bars, $\mathrm{SD} ; *, p<0.05$

\section{Discussion}

SP1-like proteins and Krüppel-like factors (KLFs) are highly related zinc-finger proteins that are important components of the eukaryotic cellular transcriptional machinery. By regulating the expression of a large number of genes containing GC-rich promoters, SP1-like/KLF transcription regulators virtually take part in all facets of cellular function, including cell proliferation, apoptosis, differentiation, and neoplastic transformation [22]. As a member of the super-family, although SP1 was mainly regarded as a basal transcription factor [6], increasing reports have indicated that SP1 is involved in the regulation of lipid metabolism, enabled by the zinc finger structures [22], which comprise a DNA-binding domain 
that also serves as a nuclear localization signal (NLS) [23]. Based on these reports, SP1 is assumed to play an important role in regulating milk fat formation. In this study, we firstly cloned the full-length cDNA of SP1 (HM_236311) from mammary gland of Xinong Saanen dairy goat. The protein, which is composed of 786 amino acid residues, contains three zinc fingers in the carboxyl terminal, similar to the other members of the super-family [22]. In addition, 2 GLN rich regions (Gln156...Gln213 and Gln352...Gln481) and an Ser/Thr rich regions, represented by SRE-rich regions, were predicted in the present study, both of which were reported to be shared among SP1-like proteins (SP1, SP2, SP3 and SP4), and predicted to be crucial for their function [22]. Thus, it is proposed that goat SP1 may share a similar function in regulating gene expression with the other members of SP family.

SP1 phosphorylation has been tied to the functional changes of DNA binding and promoter activation, which leads to expression of the target genes [24]. SP1 phosphorylation induced by oleic acid treatment led to an enhanced association with other transcription factors to synergistically increase HIV-LTR promoter activity. Further, SP1 can be phosphorylated at various sites or combination of sites, leading to a wide range of changes in SP1 function [24]. Different kinases and phosphatases targeted selected motifs to alter SP1 phosphorylation in a cell-type-specific manner [25]. The phosphorylation is predominantly on serine residues with less than $5 \%$ on threonine and none on tyrosine residues [24]. In humans the phosphorylation sites of Ser59, Ser131, Thr453, Thr579 (located in the second zinc finger) and Thr739 have been identified to be important for regulating DNA binding and gene expression. Here, we predicted 58 potential phosphorylation sites (45 serine phosphorylation sites, 10 threonine phosphorylation sites and 3 tyrosine phosphorylation sites) in the SP1 protein, including Ser59, Thr453, Thr579 and Thr739, and also found that they share a high similarity among different species (as shown in Figure S2). This indicates that goat SP1 may also play an important role in DNA promoter binding and gene expression.

In addition to phosphorylation, SP1 also contains multiple potential $O$-glycosylation sites that may be modified by $N$-acetylglucosamine residues, which has been suggested to determine the stability of cellular SP1 [26]. Reduced $O$-glycosylation of SP1 was identified to be associated with increased proteasome susceptibility in HeLa cells [26]. Additionally, the glycosylation state of SP1 is highly correlated with its ability of gene transactivation [27]. For example, $O$-GlcNAc modification is able to inhibit the interaction between SP1 peptide and TATA-binding protein-associated factor (TAF110) and holo-Sp1 in HeLa cells [28]. Corresponding to previous studies, we found 11 potential glycosylation sites in the SP1 protein, with 4 serine glycosylation sites and 7 threonine glycosylation sites, suggesting that goat SP1 may play an extensive role in milk fat metabolism regulation.

The results of tissue expression showed that $S P 1$ is mainly expressed in the tissues related to strong lipid metabolism in dairy goats, including lungs, small intestine, subcutaneous fat, mammary gland and so on, suggesting an important role in regulating lipid metabolism. However, there was no significant difference between lactation period and dry-off period. Besides, the activity of SP1 was mainly regulated by the phosphorylation level induced by stimulation from outside. This may explain the stable state of the mRNA level of SPI.

Despite the importance of SP1 in human [14] and rat [6], rare studies reported on SP1 regulated lipogenesis in ruminants' mammary gland. PPAR $\gamma$ and SREBP1 are central for milk fat synthesis regulation, and highlight a pivotal role in regulating gene expression associated with lipid metabolism [29]. LXR $\alpha$ is considered as a critical transcription factor for the activation of a number of genes [5]. PPAR $\gamma$ 
and SP1 were co-localized in the nuclei of the bovine embryo binucleated cells on Day 25 [30], and interacted with SP1 to regulate the expression of the VEGF receptor 2 [31]. The SREBP-1c promoter was accelerated by ectopic expression of SP1 and insulin further enhanced the transactivation potential of SP1. Silencing of SP1 reduced both basal and insulin-induced activation of the SREBP-1c promoter. We also found that Sp1 interacted with both SREBP-1c and LXR proteins and insulin promoted these interactions [32]. Thus, it is speculated that $S P 1$ may be involved in milk fat formation regulation via the control of these transcription factors. Here, for verification, overexpression of SP1 significantly increased the expression of PPAR $\gamma$ and $L X R \alpha$. These results were also validated by the result of siRNA treatment. However, the expression of $S R E B P 1$ remains unaffected by either overexpression or knockdown of $S P 1$, indicating its insignificant role in responses to $S P 1$ alteration. Further investigation concerning the silencing of $P P A R \gamma$ and $L X R \alpha$ may be helpful for our understanding of the mechanisms of $S P 1$ involvement in lipid metabolism regulation.

For verification of results, we detected the mRNA level of the target genes of PPAR $\gamma$ and $L X R \alpha$. The corresponding expression change of CD36, LPL ACSL1 and FABP3, all of which were reported to be downstream of PPAR $[3,29]$, and induced by the alteration of PPAR $\gamma$, confirmed that $S P 1$ may regulate fatty acid uptake (CD36 and LPL) and long-chain fatty acids transportation (ACSL1 and FABP3) via the control of expression of PPAR $\gamma$. The mutation of the $\mathrm{GC}$ region of the bovine FASN gene promoter reduced the binding of SP1 and also decreased the promoter activity [33]. ACACA and ACSS2 have been shown to be regulated by both LXR $\alpha$ and SREBP1 [34]. The alteration of ACACA in the present study was consistent with the research in bovine, in which the promoter of the ACACA gene harbors nine copies of these GC boxes known as the binding sites for the transcription factor SP1 [35]. Being considered to be the main pathway, LXR $\alpha$ can also alter lipid metabolism through other means [36]. In the present study, the expression of $A C A C A$ and ACSS 2 were changed followed the alteration of $L X R \alpha$ but not SREBP1, suggesting that $L X R \alpha$ may play an important role in mediating the function of $S P 1$ in GMECs. This hypothesis was further enhanced by the stable expression of FASN, the main target gene of SREBPI, with altered the expression of $S P 1$.

\section{Experimental Section}

\subsection{Animals and Samples Collection}

Six Xinong Saanen dairy goats (four years old, third parity) were selected and divided into two groups of three to conduct the main project (Experimental Farm of the Northwest A\&F University, Yangling, China). For one group, all three goats were slaughtered during the lactation period (100 days), tissue samples (lungs, small intestine, muscle, spleen, mammary gland, subcutaneous fat, stomach, kidney, liver and heart) were rapidly collected, and rinsed with DEPC (Sigma, St. Louis, MO, USA) sterile water, and were then used for tissue expression determination of the $S P 1$ gene [37-40]. Dry-off period was presumed from 300 days after calving to the next calving. Here, for the comparison with the expression of $S P 1$ in the lactation period, goats in the other group were slaughtered during the dry period (310 days after calving with pregnant). Mammary gland tissue samples were collected from each goat under sterile procedures to investigate the expression of SP1 in different lactation periods [41-43]. 
All samples were stored in liquid nitrogen. The Animal Care and Use Committee of the Northwest A\&F University approved all procedures and experiments.

\subsection{Total RNA Extraction and Real-Time Quantitative PCR (RT-qPCR)}

Total RNA were extracted from approximately $100 \mathrm{mg}$ tissue of slaughtered goats (listed above) using trizol reagent (Invitrogen, Shanghai, China) and treated with RNase-free DNase (CWBIO, Beijing, China) for removing DNA contamination. The concentration of RNA was analyzed with the NanoDrop 2000 (NanoDrop Technologies, Wilmington, DE, USA). The purity of RNA (A260/A280) for all samples was above 2.0. The RNA quality was verified by agarose gel electrophoresis analysis of $28 \mathrm{~S}$ and $18 \mathrm{~S}$ rRNA stained with ethidium bromide. The first-strand cDNA of various tissues were synthesized from 500 ng of purified total RNA using the PrimeScript ${ }^{\mathrm{TM}}$ RT kit (for perfect Real-time) (Takara, Otsu, Japan) according to the manufacturer's instructions. SYBR ${ }^{\circledR}$ Premix Ex Taq ${ }^{\text {TM }}$ II (Takara, Japan) was used for measuring the relative expression level of SP1 in various tissues by RT-qPCR fluorescent technology according to the manufacturer's manual on a CFX96 Real-Time PCR Detection System (Bio-Rad, Hercules, CA, USA). PCR amplification was carried out at $95^{\circ} \mathrm{C}$ for $4 \mathrm{~min}$, followed by 40 cycles of $95{ }^{\circ} \mathrm{C}$ for $15 \mathrm{~s}, 60{ }^{\circ} \mathrm{C}$ for $30 \mathrm{~s}$ and $72{ }^{\circ} \mathrm{C}$ for $30 \mathrm{~s}$. The presence of a single PCR product was verified by the dissociation protocol using incremental temperatures to $95{ }^{\circ} \mathrm{C}$ for $15 \mathrm{~s}$ plus $65{ }^{\circ} \mathrm{C}$ for $5 \mathrm{~s}$. Ubiquitously expressed transcript (UXT), mitochondrial ribosomal protein L39 (MRPL39) [44] and glyceraldehyde-3-phosphate dehydrogenase (GAPDH) [41,45] was used as an endogenous reference for normalization of targeted mRNA profiles (Table $\mathrm{S} 1$ ).

\section{3. cDNA Cloning of SP1 from Dairy Goat Mammary Gland}

The primers for SP1 cloning, as described in Table S2, were designed based on the multiple alignment of the conserved sequence of sheep (ovis, XM_004006299), bovine (Bos taurus, NM_001078027.1), human (Homo sapiens, NM_003109.1) and rattus (Rattus norvegicus, NM_013672.2). Briefly, PCRs for open reading frame (ORF) cloning were performed with an initial denaturation step at $95{ }^{\circ} \mathrm{C}$ for 5 min, 25 cycles at $94{ }^{\circ} \mathrm{C}$ for $30 \mathrm{~s}$, with $1{ }^{\circ} \mathrm{C} /$ cycle gradient temperature annealing from $50{ }^{\circ} \mathrm{C}$ for $30 \mathrm{~s}$ and extension at $72{ }^{\circ} \mathrm{C}$ for $2.5 \mathrm{~min}$. This was followed by a $10 \mathrm{~min}$ extension at $72{ }^{\circ} \mathrm{C}$. The cDNA cloning of the $5^{\prime}$ - and $3^{\prime}$-UTR were performed by nested PCR according to the manufacture's protocol of $5^{\prime}$ RACE system Ver.2.0 kit (Invitrogen, USA) and 3'-full RACE core set Ver.2.0 kit (Clontech, Takara Bio Group, Otsu, Japan), respectively. All the PCR fragments were cloned into pMD ${ }^{\circledR}$ 19-T (Takara, Otsu, Japan) vectors and sequenced by Invitrogen (Carlsbad, CA, USA).

\subsection{Sequence Analyses}

The core fragment, 3' end and 5' end sequences were assembled using the SeqMan II software in the DNAStar Package (DNAStar Inc., Madison, WI, USA) to obtain full-length cDNA of SP1. The cross-species similarities of both nucleotide and putative amino acid sequences were then calculated for homology on ClustalW (http://www.ebi.ac.uk/Tools/clustalw2/index.html). Exon information was predicted by the NCBI online tool (http://www.ncbi.nlm.nih.gov/sutils/splign/splign.cgi?textpage=online\&level=form). Protein sequence, chylomicron (MW) and isoelectric point values (PI) were analyzed by the protparam 
program of EXpASy (http://www.expasy.org/cgi-bin/protparam). ProtScale (http://us.expasy.org/cgi-bin/ protscale.pl) was used for hydrophobicity structure prediction, TMHMM (http://www.cbs.dtu.dk/ services/TMHMM/) was used for trans-membrane structure prediction, SignalP (http://www.cbs. dtu.dk/services/SignalP/) was used for signal peptide sequence prediction. Prosite program of ExPASy (http://www.expasy.org/cgi-bin/prosite/ScanView.cgi?scanfile=294556012219.scan.gz) was used for SP1 protein domains analyses. CBS netOGlyc 3.1 was used for glycosylation sites prediction (http://www.cbs.dtu.dk/services/NetOGlyc/). CBS Netphos 2.0 was used for phosphorylation sites prediction (http://www.cbs.dtu.dk/services/NetPhos/). CBS NetAcet 1.0 was used for acetylation site prediction (http://www.cbs.dtu.dk/services/NetAcet/). Phyre2 was used for protein tertiary structure prediction (http://www.sbg.bio.ic.ac.uk/ phyre2/html/page.cgi?id=index).

\section{5. siRNA Synthesis and Adenovirus Generation}

siRNA targeting SP1 and control siRNA (scramble) were designed and synthesized according to the sequence observed previously (Invitrogen, USA). siRNA sequence was as following, Sense: 5'-GCCAAUAGCUACUCAACAATT-3', anti-sense: 5'-UUGUUGAGUAGCUAUUGGCTT-3'. Control sequence was as following, sense: 5'-UUCUCCGAACGUGUCACGUTT-3', anti-sense: 5'-ACGUG ACACGUUCGGAGAATT-3'. siRNA were then transduced following the reverse transfection method described in the manual of Lipofectamine ${ }^{\mathrm{TM}}$ RNAiMAX (Invitrogen, Carlsbad, CA, USA). Adenovirus contain GFP sequence (Ad-GFP) and SP1 sequence (Ad-SP1) were generated followed the protocol described previously [45]. After proliferation, high titer adenovirus were used for transfecting GMECs.

\subsection{Cell Culture and Treatment}

HEK 293 cells were cultured in Dulbecco's modified Eagle medium (DMEM) (Hyclone, Beijing, China) compensated with 10\% fetal bovine serum (FBS) (Hyclone, Beijing, China). Mammary gland samples from three healthy dairy goats at peak lactation were collected after slaughter. GMECs purification and primary culture was according to our previous study [46]. Briefly, after slaughtering, the mammary tissues were collected and trimmed of visible fat and connective tissues and washed with D-Hank's solution several times. Acini were then selected and minced into about $1 \mathrm{~mm}^{3}$ cubes after being rinsed again with D-Hank's solution and kept up for $10 \mathrm{~min}$ at room temperature. The smaller pieces of tissues were put onto the dishes for culture. The new generated cells were then purified according to trypsin digestion method for five passages, which was presumed to remove other cells types, mainly including fibroblasts. The purified GMECs were cultured as in our previous study [47]. Briefly, GMECs were cultured in DMEM/F-12 (Hyclone, Beijing, China), compensated with insulin (5 mg/L, Sigma, USA), hydrocortisone (5 mg/L, Sigma, USA), penicillin/streptomycin (10 kU/L, Harbin Pharmaceutical Group, Harbin, China), epidermal growth factor $(10 \mathrm{ng} / \mathrm{mL}$, Invitrogen, Carlsbad, CA, USA) and FBS (10\%). The cultured GMECs were evaluated by growth curve fitting, karyotype analysis, immunofluorescence staining (keratin, epithelial membrane antigen (EMA), vimentin, $\beta$-casein), oil red staining and RT-PCR of the $\beta$-casein gene and found that the methods we used in our group can obtain suitable GMECs with the function of secretion [46]. Prior to experiments, cells were changed to lipid-free, serum-free medium (basal medium plus $1 \mathrm{~g} / \mathrm{L}$ bovine serum albumin; BSA) supplemented with prolactin $(1.5 \mathrm{mg} / \mathrm{L})$ to promote lactogenesis [48]. 
GMECs at $70 \%-80 \%$ confluence were transduced with adenovirus supernatant at a multiplicity of infection (MOI) of 200. Ad-GFP was used as the control. Cells were harvested for RNA extraction after transduction for $72 \mathrm{~h}$ using Cell RNA Extraction Kit (TIANGEN, Beijing, China).

\subsection{Western Blot Analyses}

The protein samples were extracted with radio immunoprecipitation assay lysis buffer (RIPA) (Solarbio, Beijing, China) supplemented with 10\% PMSF. Western blot was assessed following the protocol described previously [49]. Polyclonal rabbit anti-SP1 (Abcam, ab13370, Cambridge, UK, 1:3000) and monoclonal mouse anti-GAPDH (CWBIO, China, 1:1000) were used as the primary antibodies. Polyclonal goat anti-mouse/rabbit IgG coupled to HRP (TIANGEN, AB101, China, and 1:1000) was used as secondary antibody respectively. Signals were detected using the chemiluminescent ECL Western blot detection system (Pierce, Thermo Fisher Scientific Inc., Rockford, IL, USA).

\subsection{Statistical Analyses}

The results are expressed as mean \pm SD, and SPSS 19.0 (IBM, Armonk, NY, USA) was used for statistical analysis. Data of qPCR were analyzed relative to the control using the $2^{-\Delta \Delta C t}$ method. Group data for multiple comparisons were analyzed by ANOVA using a general linear model procedure followed by Tukey's test. Significance was established at a $p<0.05$.

\section{Conclusions}

In summary, we firstly cloned 4376 bp full-length cDNA of the $S P 1$ gene, includes 103 bp of 5'UTR, 2358 bp of ORF (HM_236311) and 1915 bp of 3'UTR using a RACE method. Goat SP1 is predominantly expressed in the lungs and small intestine, followed by muscle, spleen, mammary gland and subcutaneous fat. Lactation stages have no significant effects on $S P 1$ expression. Over expression of $S P 1$ in GMECs led to higher concentration of $P P A R \gamma$ and lower $L X R \alpha$ mRNA levels in GMECs, and correspondingly altered the expression of CD36, LPL, ACSL1, FABP 3, ACACA and ACSS2. These results were further enhanced by the silencing of $S P 1$. The expression of $S R E B P 1$ and FASN remained unaffected by the alteration of SP1 mRNA. These results indicate that SP1 may be involved in milk fat metabolism via the control of PPAR $\gamma$ and $\operatorname{LXR} \alpha$.

\section{Supplementary Materials}

Supplementary materials can be found at http://www.mdpi.com/1422-0067/16/01/1806/s1.

\section{Acknowledgments}

This research was jointly supported by the "National Natural Science Foundation of China (31372281)", the "Transgenic New Species Breeding Program of China (2014ZX08009-051B)", "Special Fund for Agro-scientific Research in the Public Interest (201103038)" and "Guangxi Key Laboratory of Animal Genetic Improvement of Open Project” (2014GXKLLGI). 


\section{Author Contributions}

Jiangjiang Zhu and Yuting Sun designed and performed the in vitro cell culture and qPCR; Yanhong Cao, $\mathrm{Min} \mathrm{Wu}$, and Jianhua Li performed all the gene cloning and computational studies; Jiangjiang Zhu reviewed the data and drafted the manuscript; Jun Luo checked, revised and finalized the paper.

\section{Conflicts of Interest}

The authors declare no conflict of interest.

\section{References}

1. Haenlein, G.F.W. Goat milk in human nutrition. Small Rumin. Res. 2004, 51, 155-163.

2. Williams, C. Dietary fatty acids and human health. Ann. Zootech. 2000, 49, 165-180.

3. Shi, H.; Luo, J.; Zhu, J.; Li, J.; Sun, Y.; Lin, X.; Zhang, L.; Yao, D.; Shi, H. PPARG regulates genes involved in triacylglycerol synthesis and secretion in mammary gland epithelial cells of dairy goats. PPAR Res. 2013, 2013, 310948:1-310948:10.

4. Harvatine, K.J.; Boisclair, Y.R.; Bauman, D.E. Recent advances in the regulation of milk fat synthesis. Animal 2009, 3, 40-54.

5. Oppi-Williams, C.; Suagee, J.K.; Corl, B.A. Regulation of lipid synthesis by liver X receptor a and sterol regulatory element-binding protein 1 in mammary epithelial cells. J. Dairy Sci. 2013, 96, 112-121.

6. Solomon, S.S.; Majumdar, G.; Martinez-Hernandez, A.; Raghow, R. A critical role of sp1 transcription factor in regulating gene expression in response to insulin and other hormones. Life Sci. 2008, 83, 305-312.

7. Huang, C.; Xie, K. Crosstalk of $\mathrm{Sp} 1$ and Stat3 signaling in pancreatic cancer pathogenesis. Cytokine Growth Factor Rev. 2012, 23, 25-35.

8. Safe, S.; Abdelrahim, M. Sp transcription factor family and its role in cancer. Eur. J. Cancer 2005, 41, 2438-2448.

9. Bouwman, P.; Philipsen, S. Regulation of the activity of Sp1-related transcription factors. Mol. Cell. Endocrinol. 2002, 195, 27-38.

10. Gill, G.; Pascal, E.; Tseng, Z.H.; Tjian, R. A glutamine-rich hydrophobic patch in transcription factor SP1 contacts the dTAFII110 component of the drosophila TFIID complex and mediates transcriptional activation. Proc. Natl. Acad. Sci. USA 1994, 91, 192-196.

11. Hoey, T.; Weinzierl, R.O.; Gill, G.; Chen, J.L.; Dynlacht, B.D.; Tjian, R. Molecular cloning and functional analysis of drosophila TAF110 reveal properties expected of coactivators. Cell 1993, 72 , 247-260.

12. Liu, W.L.; Coleman, R.A.; Grob, P.; King, D.S.; Florens, L.; Washburn, M.P.; Geles, K.G.; Yang, J.L.; Ramey, V.; Nogales, E.; et al. Structural changes in TAF4b-TFIID correlate with promoter selectivity. Mol. Cell 2008, 29, 81-91.

13. Jeon, B.-N.; Kim, Y.-S.; Choi, W.-I.; Koh, D.-I.; Kim, M.-K.; Yoon, J.-H.; Kim, M.-Y.; Hur, B.; Paik, P.D.-H.; Hur, M.-W. Kr-pok increases FASN expression by modulating the DNA binding of SREBP-1c and Sp1 at the proximal promoter. J. Lipid Res. 2012, 53, 755-766. 
14. Lu, S.; Archer, M.C. Sp1 coordinately regulates de novo lipogenesis and proliferation in cancer cells. Int. J. Cancer 2010, 126, 416-425.

15. Fukuda, H.; Iritani, N.; Noguchi, T. Transcriptional regulatory regions for expression of the rat fatty acid synthase. FEBS Lett. 1997, 406, 243-248.

16. Reed, B.D.; Charos, A.E.; Szekely, A.M.; Weissman, S.M.; Snyder, M. Genome-wide occupancy of SREBP1 and its partners NFY and SP1 reveals novel functional roles and combinatorial regulation of distinct classes of genes. PLoS Genet. 2008, 4, e1000133.

17. Alberts, A.W.; Vagelos, P.R. Acetyl CoA carboxylase. I. Requirement for two protein fractions. Proc. Natl. Acad. Sci. USA 1968, 59, 561-568.

18. Moon, Y.A.; Kim, K.S.; Cho, U.H.; Yoon, D.J.; Park, S.W. Characterization of regulatory elements on the promoter region of human ATP-citrate lyase. Exp. Mol. Med. 1999, 31, 108-114.

19. Daniel, S.; Kim, K.H. Sp1 mediates glucose activation of the acetyl-CoA carboxylase promoter. J. Biol. Chem. 1996, 271, 1385-1392.

20. Krey, G.; Mahfoudi, A.; Wahli, W. Functional interactions of peroxisome proliferator-activated receptor, retinoid-X receptor, and $\mathrm{Sp} 1$ in the transcriptional regulation of the acyl-coenzyme-A oxidase promoter. Mol. Endocrinol. 1995, 9, 219-231.

21. Schmitz, G.; Langmann, T. Transcriptional regulatory networks in lipid metabolism control ABCA1 expression. Biochim. Biophys. Acta 2005, 1735, 1-19.

22. Kaczynski, J.; Cook, T.; Urrutia, R. Sp1- and kruppel-like transcription factors. Genome Biol. 2003, 4, 206. doi:10. 1186/gb-2003-4-2-206.

23. Ito, T.; Azumano, M.; Uwatoko, C.; Itoh, K.; Kuwahara, J. Role of zinc finger structure in nuclear localization of transcription factor Sp1. Biochem. Biophys. Res. Commun. 2009, 380, 28-32.

24. Chu, S.; Ferro, T.J. Sp1: Regulation of gene expression by phosphorylation. Gene 2005, 348, 1-11.

25. Samson, S.L.; Wong, N.C. Role of Sp1 in insulin regulation of gene expression. J. Mol. Endocrinol. 2002, 29, 265-279.

26. Han, I.; Kudlow, J.E. Reduced O glycosylation of $\mathrm{Sp} 1$ is associated with increased proteasome susceptibility. Mol. Cell. Biol. 1997, 17, 2550-2258.

27. Wells, L.; Vosseller, K.; Hart, G.W. Glycosylation of nucleocytoplasmic proteins: Signal transduction and O-GlcNAc. Science 2001, 291, 2376-2378.

28. Roos, M.D.; Su, K.; Baker, J.R.; Kudlow, J.E. O glycosylation of an Sp1-derived peptide blocks known Sp1 protein interactions. Mol. Cell. Biol. 1997, 17, 6472-6480.

29. Bionaz, M.; Loor, J. Gene networks driving bovine milk fat synthesis during the lactation cycle. BMC Genomics 2008, 9, 366, doi:10.1186/1471-2164-9-366.

30. Degrelle, S.A.; Murthi, P.; Evain-Brion, D.; Fournier, T.; Hue, I. Expression and localization of DLX3, PPARG and Sp1 in bovine trophoblast during binucleated cell differentiation. Placenta 2011, 32, 917-920.

31. Sassa, Y.; Hata, Y.; Aiello, L.P.; Taniguchi, Y.; Kohno, K.; Ishibashi, T. Bifunctional properties of peroxisome proliferator-activated receptor $\gamma 1$ in KDR gene regulation mediated via interaction with both Sp1 and Sp3. Diabetes 2004, 53, 1222-1229. 
32. Deng, X.; Yellaturu, C.; Cagen, L.; Wilcox, H.G.; Park, E.A.; Raghow, R.; Elam, M.B. Expression of the rat sterol regulatory element-binding protein-1c gene in response to insulin is mediated by increased transactivating capacity of specificity protein 1 (Sp1). J. Biol. Chem. 2007, 282, 17517-17529.

33. Ordovas, L.; Roy, R.; Pampin, S.; Zaragoza, P.; Osta, R.; Rodriguez-Rey, J.C.; Rodellar, C. The g.763G $>$ C SNP of the bovine FASN gene affects its promoter activity via Sp-mediated regulation: Implications for the bovine lactating mammary gland. Physiol. Genomics 2008, 34, 144-148.

34. McFadden, J.W.; Corl, B.A. Activation of liver X receptor (LXR) enhances de novo fatty acid synthesis in bovine mammary epithelial cells. J. Dairy Sci. 2010, 93, 4651-4658.

35. Mao, J.; Seyfert, H.M. Promoter II of the bovine acetyl-coenzyme A carboxylase- $\alpha$-encoding gene is widely expressed and strongly active in different cells. Biochim. Biophys. Acta 2002, 1576, 324-329.

36. Liang, G.; Yang, J.; Horton, J.D.; Hammer, R.E.; Goldstein, J.L.; Brown, M.S. Diminished hepatic response to fasting/refeeding and liver $\mathrm{X}$ receptor agonists in mice with selective deficiency of sterol regulatory element-binding protein-1c. J. Biol. Chem. 2002, 277, 9520-9528.

37. Zhao, W.S.; Hu, S.L.; Yu, K.; Wang, H.; Wang, W.; Loor, J.; Luo, J. Lipoprotein lipase, tissue expression and effects on genes related to fatty acid synthesis in goat mammary epithelial cells. Int. J. Mol. Sci. 2014, 15, 22757-22771.

38. Li, Z.; Lan, X.; Guo, W.; Sun, J.; Huang, Y.; Wang, J.; Huang, T.; Lei, C.; Fang, X.; Chen, H. Comparative transcriptome profiling of dairy goat micrornas from dry period and peak lactation mammary gland tissues. PLoS One 2012, 7, e52388.

39. Wang, A.; Gu, Z.; Heid, B.; Akers, R.M.; Jiang, H. Identification and characterization of the bovine G protein-coupled receptor GPR41 and GPR43 genes. J. Dairy Sci. 2009, 92, 2696-2705.

40. Zhang, Y.; Zhang, X.D.; Liu, X.; Li, Y.S.; Ding, J.P.; Zhang, X.R.; Zhang, Y.H. Reference gene screening for analyzing gene expression across goat tissue. Asian-Australas. J. Anim. Sci. 2013, 26, 1665-1671.

41. Lin, X.-Z.; Luo, J.; Zhang, L.-P.; Wang, W.; Shi, H.-B.; Zhu, J.-J. miR-27a suppresses triglyceride accumulation and affects gene mRNA expression associated with fat metabolism in dairy goat mammary gland epithelial cells. Gene 2013, 521, 15-23.

42. Li, J.; Luo, J.; Wang, H.; Shi, H.; Zhu, J.; Sun, Y.; Yu, K.; Yao, D. Adipose triglyceride lipase regulates lipid metabolism in dairy goat mammary epithelial cells. Gene 2015, 554, 125-130.

43. Zhu, J.J.; Luo, J.; Wang, W.; Yu, K.; Wang, H.B.; Shi, H.B.; Sun, Y.T.; Lin, X.Z.; Li, J. Inhibition of FASN reduces the synthesis of medium-chain fatty acids in goat mammary gland. Animal 2014, 8, 1469-1478.

44. Kadegowda, A.K.; Bionaz, M.; Thering, B.; Piperova, L.S.; Erdman, R.A.; Loor, J.J. Identification of internal control genes for quantitative polymerase chain reaction in mammary tissue of lactating cows receiving lipid supplements. J. Dairy Sci. 2009, 92, 2007-2019.

45. Shi, H.B.; Luo, J.; Yao, D.W.; Zhu, J.J.; Xu, H.F.; Shi, H.P.; Loor, J.J. Peroxisome proliferator-activated receptor- $\gamma$ stimulates the synthesis of monounsaturated fatty acids in dairy goat mammary epithelial cells via the control of stearoyl-coenzyme A desaturase. J. Dairy Sci. 2013, $96,7844-7853$. 
46. Shi, H.; Shi, H.; Luo, J.; Wang, W.; Haile, A.B.; Xu, H.; Li, J. Establishment and characterization of a dairy goat mammary epithelial cell line with human telomerase (ht-MECs). Anim. Sci. J. 2014, $85,735-743$.

47. Wang, W.; Luo, J.; Zhong, Y.; Lin, X.-Z.; Shi, H.-B.; Zhu, J.-J.; Li, J.; Sun, Y.-T.; Zhao, W.-S. Goat liver $\mathrm{X}$ receptor $\alpha$, molecular cloning, functional characterization and regulating fatty acid synthesis in epithelial cells of goat mammary glands. Gene 2012, 505, 114-120.

48. Peterson, D.G.; Matitashvili, E.A.; Bauman, D.E. The inhibitory effect of trans-10, cis-12 CLA on lipid synthesis in bovine mammary epithelial cells involves reduced proteolytic activation of the transcription factor SREBP-1. J. Nutr. 2004, 134, 2523-2527.

49. Kang, Y.; Hengbo, S.; Jun, L.; Jun, L.; Wangsheng, Z.; Huibin, T.; Huaiping, S. PPARG modulated lipid accumulation in dairy gmec via regulation of ADRP gene. J. Cell. Biochem. 2015, 116, 192-201.

(C) 2015 by the authors; licensee MDPI, Basel, Switzerland. This article is an open access article distributed under the terms and conditions of the Creative Commons Attribution license (http://creativecommons.org/licenses/by/4.0/). 